\title{
ISOKINETIC ASSESSMENT OF THE FOREARM AND WRIST MUSCLES
}

B. FORTHOMME, J.L. CROISIER, M. FOIDART-DESSALLE, J.M. CRIELAARD Department of Physical Medicine, CHU Sart Tilman, Liege, Belgium 


\section{Introduction}

Upper extremity insults caused by single stress or by repetitive microtrauma occur in a large variety of sport or professional activities [1,21,22,32,24,3]. Among these injuries, lateral epicondylitis could result from excessive use of the wrist extensor and forearm supinator muscles $[14,27,7,12,13]$.

Isokinetics has been increasingly used for strength measurement of muscle groups with tendon disorders with the aim of interpreting the mechanical profile of the muscle and to assess treatment effects $[7,15,16]$. Dynamometers also allow eccentric training in patients with chronic tendinitis $[7,17,34]$. Nevertheless, the ability to accurately assess muscular strength in testing situations appears fundamental and normative data remain vital in order to interpret pathological cases, particularly of a unilateral type [31,28,25,30].

Relatively little is known about isokinetic profiles of the wrist muscles. Kramer et al. [26] examined the reliability of isometric pronation and supination measurements using two dynamometers. Friedman [15] compared isokinetic peak torques in symptomatic and asymptomatic lateral epicondyles of women using a single speed test protocol (concentric $120^{\circ} . \mathrm{s}^{-1}$ mode). Frobose et al. [16] tested the wrist extensors and flexors in the concentric mode. Nevertheless, no comprehensive isokinetic assessment of the forearm and wrist muscles (in the concentric and eccentric modes through low and high speeds) has been previously, particularly emphasizing reproducibility.

The primary aim of the study was therefore to establish reference concentric and eccentric strength values related to the forearm (pronators - supinators) and wrist (flexors - extensors) muscles and their agonist/antagonist ratios. The inter-session reproducibility of measurements, the effect of weight, side of testing, dominance and gender on torques were also investigated. 


\section{Material and methods}

\subsection{Subjects}

Forty healthy subjects (20 women : $23 \pm 3$ years, $59.5 \pm 8.5 \mathrm{~kg}$ and 20 men : $23 \pm 3$ years, 75 $\pm 7 \mathrm{~kg}$ ) with moderate occupational and spare-time activity level participated in the study. Among them, 39 were right-hand dominant. None of them had previously presented orthopedic or neurologic deficiencies of the upper extremity.

\subsection{Experimental procedure}

Measurements were undertaken using a Cybex Norm dynamometer. For the forearm pronator (PRO) and supinator (SUP) muscles, subjects were seated in the testing chair in front of the dynamometer, the forearm in a neutral position supported on an horizontal plane (Fig. 1). The axis of the dynamometer was aligned with the forearm and the elbow was maintained at $60^{\circ}$ of flexion. The range of motion was fixed at $160^{\circ}$ corresponding to $80^{\circ}$ of supination and $80^{\circ}$ of pronation. No gravity correction was performed. Prior to testing, an adequate familiarization with the dynamometer was provided in the form of warm-up concentric isokinetic repetitions at the angular speed of $60^{\circ} / \mathrm{s}$. Three preliminary repetitions routinely preceded each test speed. The assessment protocol included concentric exertions at the successive angular velocities of $90 \%$ s (4 repetitions) and $30 \%$ s (4 repetitions). Thereafter, one eccentric angular speed of $60 \%$ (4 repetitions) was applied.

Wrist flexor (FLE) and extensor (EXT) muscles assessment was conducted with the subjects seated, the forearm supported on an horizontal plane and the elbow maintained flexed at $60^{\circ}$. The joint center of the wrist was aligned with the rotational axis of the dynamometer's motor and the forearm was placed in a pronation position (Fig. 2). Testing was performed along a constant range of motion of $140^{\circ}$ on both sides of neutral $\left(-70^{\circ}\right.$ to $\left.+70^{\circ}\right)$. No gravity was performed. The assessment protocol consisted of $90 \%$ s (4 repetitions) and 30\% (4 repetitions) angular velocities in the concentric mode and $60 \%$ s (4 repetitions) in the eccentric mode. 
The analyzed parameters included:

- $\quad$ absolute peak-torque (PT in N.m) and bodyweight normalized peak-torque (in N.m. $\mathrm{kg}^{-1}$ );

- bilateral comparison permitting determination of asymmetries (expressed in \%) and a possible dominance effect;

- conventional pronator/supinator (PRO/SUP) and flexor/extensor (FLE/EXT) ratios established for the same mode of contraction.

Furthermore we have studied:

- the coefficients of variation calculated on peak torques in a 10 days test-retest session based on 10 subjects who were randomly selected

- the gender differences in bodyweight normalized torque values

\subsection{Statistical methods}

For each variable, differences were evaluated using a paired and simple Student t test. The level of significance was set at $\mathrm{p}=0.05$. Reproducibility was assessed by the means of coefficients of variation (CV).

$$
C V=\frac{S}{\bar{m}} \times 100 \%, \quad S=\sqrt{\frac{\sum d^{2}}{2 n}}
$$

\footnotetext{
$\mathbf{C V}=$ coefficient of variation,

$\overline{\mathbf{m}}=$ mean values of absolute peak torques,

$\mathbf{d}=$ difference between peak torque values for the two sessions,

$\mathbf{n}=$ number of subjects.
} 


\section{Results}

\section{Reproducibility}

The coefficients of variation (CV) calculated based on the peak torques in the test-retest procedure are presented in Table 1.

\section{Forearm pronator - supinator muscle groups}

Means and standard deviations (SD) for forearm pronator and supinator absolute PT (in N.m), bodyweight normalized PT (in N.m. $\mathrm{kg}^{-1}$ ) and the mean values of the PRO/SUP ratios are respectively displayed for males and females in Tables 2-4. Maximal concentric torque significantly decreased with increasing movement velocity. The eccentric contraction mode showed the highest strength development for both muscle groups (Tables 2 and 3). The eccentric ratio (Table 4) was in most cases smaller than the classical concentric ratio ( $\mathrm{p}$ 0.05). The concentric ratios were not modified by the speed used in assessment. A significant difference was occasionally demonstrated between the dominant (D) and the non-dominant (ND) sides (Tables 2-4).

Ignoring dominance, bilateral differences throughout concentric contractions ranged from:

- $\quad$ in pronation $9( \pm 9) \%$ to $14( \pm 13) \%$ (men) and $15( \pm 13) \%$ to $24( \pm 9) \%$ (women)

- $\quad$ in supination, $17,5( \pm 13) \%$ to $18.5( \pm 9) \%$ (men) and $19( \pm 16)$ to $20( \pm 11) \%$ (women)

The eccentric mode indicated bilateral differences of between $13( \pm 12) \%$ and $18( \pm 13) \%$ for forearm assessment. The magnitude of the standard deviations observed in our study demonstrated a conspicuous between-subject variations.

The average PRO/SUP concentric torque ratio (Table 4) values ranged between $0.94( \pm 0.23)$ and 1.45 ( \pm 0.42) indicating superiority of the pronators relative to the supinators. In the eccentric mode, the ratios ranged from $0.92( \pm 0.18)$ to $1.08( \pm 0.36)$. In spite of using bodyweight normalization, men appeared stronger than women in most of the testing conditions $(\mathrm{p}<0.05)$. Nevertheless, men and women were characterized by similar ratios. 


\section{Wrist flexor-extensor muscle groups}

The mean absolute and bodyweight normalized PT ( \pm SD) developed by flexor and extensor muscles of the wrist are outlined for men and women respectively in Tables 5 and 6 . The mean values $( \pm \mathrm{SD})$ of FLE/EXT ratios are presented in Table 7. Similar to the forearm muscles, the wrist muscles strength profile was in line with the force-velocity relationship. The concentric ratio (Table 7) was not modified by the angular velocity and the eccentric ratio seemed smaller $(\mathrm{p}<0.05)$ than the concentric value at $90 \%$ s.

In the male population, flexor muscles of the dominant wrist (Table 5) appeared systematically stronger compared to the non-dominant side $(\mathrm{p}<0.05)$. Consequently, the FLE/EXT ratio (Table 7) remained stronger for the dominant side in all assessment conditions. In the female group, the only significant difference $(\mathrm{p}<0.05)$ was noted between the dominant and the no-dominant sides with respect to the flexor muscles in the concentric mode at $30 \%$ s allowing a significant rise of the dominant ratio for that speed (Tables 6,7 ).

Bilateral concentric PT comparison, excluding the dominant influence, showed a difference ranging from:

- in flexion, $18( \pm 12) \%$ to $22.5( \pm 10) \%$ (men) and $16.5( \pm 11) \%$ to $18( \pm 14) \%$ (women)

- in extension, $13( \pm 6) \%$ to $14( \pm 9) \%$ (men) and $15.5( \pm 14) \%$ to $20( \pm 12.24) \%$ (women)

Regarding the eccentric mode, the bilateral asymmetries indicated a difference of between 13 ( \pm 9$) \%$ to 21 ( \pm 10$) \%$ for the wrist muscles. These drastic bilateral differences were due to inter-individual variations.

The FLE/EXT concentric PT ratios (Table 7) were between $2.55( \pm 0.29)$ and $1.80( \pm 0.51)$. In the eccentric mode, ratios reached $1.23( \pm 0.28)$ and $2.02( \pm 0.53)$. These results indicate that flexor muscles are stronger than the extensors in all assessment conditions.

Men's PT values appeared higher than the corresponding values in women in the following conditions:

- flexor muscles of the dominant side in all testing conditions;

- flexor muscles of the non-dominant side for concentric mode at $90 \% \mathrm{~s}$ 
The men's ratio in the eccentric mode remained significantly higher in comparison with the women's values.

\section{Discussion}

Despite the growing popularity of isokinetic testing [10], knee represents the most common joint assessed. Nevertheless, clinicians and researchers are becoming increasingly interested in isokinetic assessment and exercise of upper extremity joints as the shoulder and the elbow $[2,23,30,29]$. Recent studies have investigated distal muscles of the upper limb, particularly forearm and wrist muscles $[15,16,18,23,26]$. Some pathologies which result from sport activities and specific professional occupations involve these muscles $[32,12,13,3]$. In our Center, we are specifically challenged with patients suffering from chronic epicondylitis $[14,7]$.

Quantitative assessment of forearm and wrist muscle performance can add substantially to the understanding of elbow function in patients with disorders: Isokinetic evaluation enables detection of bilateral asymmetries and agonist-antagonist imbalances which provides an interesting venue for exploring these musculo-skeletal injuries [4,25]. Moreover, accurate and precise muscular strength measurement is fundamental in testing situation $[31,28,25,30]$. A wide variety of test protocols exists with a spectrum of slow and fast isokinetic velocities. The testing modalities we propose were determined on the following basis:

- in isokinetic exercise, excessively low speeds frequently provoke discomfort, justifying an inferior limit of $30 \%$ s in the concentric mode 
- concentric velocities higher than $90 \%$ s drastically reduce the isokinetic sector. Some authors $[18,15]$ have used $120 \%$ s or even $180 \%$ s although in our practical experience, subjects showed great difficulties in reaching such velocities. Also, the velocity of $90^{\circ} / \mathrm{s}$ seemed to facilitate motor learning;

- a single eccentric test velocity was used as this contraction mode plays a specific role in some fields of rehabilitation $[14,7,17,34]$. At the same time, the eccentric contraction is regularly cited as a likely causal factor of muscle and tendon injuries [4]. Despite this fact, few authors have incorporated eccentric testing in wrist and forearm isokinetic assessment $[14,7,17,34]$. We limited the eccentric protocol to one speed test because of the motor learning difficulties at higher eccentric speed for sedentary subjects.

The absolute reliability in repeated tests procedure of peak torque measurement for the forearm muscles appeared satisfactory with homogeneous coefficients of variation (CV) inferior or equal to $12 \%$ and excellent for the wrist muscles with a CV ranged from $5 \%$ to $9.4 \%$. No significant difference was shown between D and ND reproducibility. In comparison, in knee assessment the CV is typically less than $10 \%$ throughout concentric and eccentric actions [5]. The lower maximal absolute strength developed by forearm and wrist musculature means that a small change in torque greatly influenced the coefficient of variation and the sensitivity level of the dynamometer (working by step of 1 N.m), contributing to a rise in $\mathrm{CV}$ values. The $\mathrm{CV}$ tended to be lower during assessment in the concentric mode at the lowest speed than for the higher concentric speed or the eccentric mode despite adequate warm-up and familiarization. In such modalities, subjects seemed to have increased motor control difficulty [30]. The lowest concentric speed on extensor 
muscles represented a special feature with a higher CV due to specific difficulties to control compensations throughout the movement.

Among the muscles evaluated in our study the wrist flexors were found to be the strongest group followed by extensors, pronators and supinators respectively. The torques developed by the upper extremity muscle groups appear weaker in comparison to the knee performances: the quadriceps commonly develop $2.5 \mathrm{~N} . \mathrm{m}^{\mathrm{kg}}{ }^{-1}$ in the concentric mode at $60^{\circ} . \mathrm{s}^{-1}$ in sedentary males [5].

Regarding sex differences men were stronger than women (mainly on the dominant side). On average, the strength ratios women/men, established with respect to the dominant side were: $58 \%$ (55-63 \%) for the pronators, $61 \%$ (57-63 \%) for the supinators, $57 \%$ (55-60 \%) for the flexors and $73 \%$ [62-90 \%] for the extensors. These results are in very close agreement with those reported by Dvir et al. [11] relating to trunk strength. An exception was noted with respect to wrist extensors in women. On the other hand, the reciprocal strength ratios which indicate muscular balance around the joint [25] were generally independent of sex. There was only one exception: the FLE/EXT ratios in the eccentric mode which was greater for the male dominant $(\mathrm{p}<0.001)$ and non-dominant $(\mathrm{p}<0.05)$ sides. Indeed, the men's flexors demonstrated superiority in all conditions but their extensor muscles remained inferior in comparison with female relative values in the eccentric mode.

Based on the physiological force-velocity principle $[8,35,20]$ concentric peak torques decreased with the rise in speed and the eccentric torque remained highest for all muscle assessments. In our study, concentric ratios were not influenced by the angular speed even though the eccentric ratio was significantly $(p<0.05)$ reduced in comparison to the concentric 
value at $90^{\circ} \cdot \mathrm{s}^{-1}$. The reduced eccentric ratio could be due to a different braking behavior of the flexors compared to the extensors as well as between the pronators and the supinators. Incidentally we assume that, in a population practicing sports activity, this eccentric ratio could be quite different with regard to the specific activity [13,3].

In concentric exertions and excluding the influence of dominance, the bilateral asymmetries did not exceed $24( \pm 9) \%$, observed on the pronators $(90 \%$ s). Referring to the knee profile, bilateral differences remained less than $10 \%$ [5]. The low level of PT developed for the upper distal extremity as well as inter-individual differences may have contributed to these results.

In order to determine the limit between normal or abnormal results in side-to-side comparison it is necessary to integrate the common bilateral asymmetry results and the CV values. Sapega [33] considered the following general guideline in knee side-to-side comparison: strength imbalances of less than $10 \%$ are considered normal; differences of 10-20\% possibly abnormal and those greater than $20 \%$ probably abnormal. Nevertheless, in the current study wrist and forearm bilateral differences in excess of $20 \%$ were observed. In the absence of an indication of some pathology, we conclude that a $20 \%$ difference may be normal for these muscle groups.

The possible influence of the dominant side must also be considered in such a bilateral comparison. Comparison of the average peak torque data between the dominant and nondominant sides revealed significant differences for the dominant flexor $(p<0.005)$ and supinator muscles $(\mathrm{p}<0.05)$ in the male population. These upper limb muscle groups are more frequently implicated in various daily living activities (gardening, lifting tasks). We 
speculate a possible increased effect of dominance in some sports activities particularly overhead throwing $[19,29]$ that require participation of these muscles. As a result, the ratios showed a dominance effect mainly for the male FLE/EXT ratio due to a higher torque developed by the dominant flexors compared to lower extensor muscle adaptation.

Commonly, concentric and eccentric PT ratios (PRO/SUP, FLE/EXT) reflected the superiority of the muscles (PRO - FLE) in comparison to the opposite groups (SUP - EXT). The absence of gravity compensation for the wrist and forearm assessment (using a Cybex Norm dynamometer) and the adapted forearm installation in a pronation position could overestimate the flexor group during the wrist strength measurement. Calmels et al. [2] reported similar ratios in spite of a different installation (forearm in a neutral position), probably due to the population recruited (tennis players) and the dynamometer employed. 


\section{Conclusion}

Objective and accurate measurement of strength may be helpful in assessing function of the normal and pathologic upper limb muscles and joints. Forearm and wrist muscles have attracted little attention although they are highly involved in a large number of sport and professional activities. The purpose of this study was to investigate specific concentric and eccentric strength profiles of forearm and wrist muscles in order to obtain reliable reference data in normal male and female groups. We obtained a satisfactory test-retest reproducibility with a CV of less than $12 \%$. Men are commonly stronger than women, mainly on the dominant side. The PRO-SUP ratio reached 1.45 on the concentric mode and 1.08 on the eccentric mode whereas the FLE/EXT ratio reached 2.55 in the concentric mode and 2.02 in the eccentric contraction. For all muscle groups studied the bilateral asymmetries remained less than $24 \%$ indicating a cutoff of $20 \%$ for distinguishing pathologic from normal bilateral asymmetry. The male group showed dominant effect, mainly for the flexor and the supinator muscles. These results can be usefull in order to characterize a pathological context in clinical situations. 


\section{REFERENCES}

[1] L.C. Almekinders and S.V. Almekinders, Outcome in the treatment of chronic overuse sports injuries: A retrospective study, J Orthop Sports Phys Ther 19 (1994), 157-161.

[2] P. Calmels, G. Abeillon and M. Domenach et al., Bilan isocinétique du coude et du poignet, in: Isocinétisme et médecine de rééducation, Masson, Paris, 1991, pp. 66-74.

[3] W.P. Cooney, Sports injuries to the upper extremity. How to recognize and deal with some common problems, Postgrad Med 76 (1984), 45-50.

[4] J.L. Croisier and J.M. Crielaard, Hamstring muscle tear with recurrent complaints: an isokinetic profile, Isokinetics Exerc Sci 8 (2000), 175-180.

[5] J.L. Croisier and J.M. Crielaard, Méthodes d'exploration de la force musculaire : une analyse critique, Ann Réadaptation Méd Phys 42 (1999), 311-322.

[6] J.L. Croisier, Contribution fondamentale et clinique à l'exploration musculaire isocinétique, Ph. D. Dissertation, University of Liege, 1996.

[7] J.L. Croisier, B. Forthomme and M. Foidart-Dessalle et al., Treatment of recurrent tendinitis by isokinetic eccentric exercises, Isokinetics Exerc Sci 9 (2001), 133-141.

[8] J.L. Croisier, G. Camus and M. Ledent et al., La relation force-vitesse étudiée en isocinétisme, Arch Physiol Biochem 106B (1998), 70.

[9] S. Curwin, Acute sports injuries: The aetiology and treatment of tendinitis, in: Oxford Textbook of Sports Medicine, M. Harries, C. Williams, W.D. Stanish and L.J. Micheli, Oxford Medical Publications, Oxford University Press, 1994, pp. 512-528.

[10] Z. Dvir, The clinical applicability of isokinetics: a review, Clin Biomech 6 (1991), 133-144. 
[11] Z. Dvir, J. Keating, Reproducibility of concentric and eccentric isokinetic trunk extension strength using short range of motion protocol. Clinical Biomechanics $\mathbf{1 6}$ (2001), 627-30.

[12] L.D. Field and D.W. Altchek, Elbow injuries, Clin Sports Med 14 (1995), 59-78.

[13] L.D. Field and F.H. Savoie, Common elbow injuries in sport, Sports Med 26 (1998), 193-205.

[14] B. Forthomme, J.L. Croisier and M. Foidart et al., Exploration isocinétique des muscles de l'avant-bras et du poignet. Application à une pathologie tendineuse, (in press) (2002).

[15] P.J. Friedman, Isokinetic peak torque in women with unilateral cumulative trauma disorders and healthy control subjects, Arch Phys Med Rehabil 79 (1998), 816-819.

[16] I. Frobose, M. Settner and H. Maier, Analysis of strength values of the wrist muscles and its clinical relevance, Zeitschrift für Orthopadie und Ihre Grenzgebiete 133 (1995), 79-83.

[17] I. Fyfe and W.D. Stanish, The use of eccentric training and stretching in the treatment and prevention of tendon injuries, Clin Sports Med 11 (1992), 601-624.

[18] M.A. Gallagher, F. Cuomo and L. Polonsky et al., Effects of age, testing speed, and arm dominance on isokinetic strength of the elbow, J Shoulder Elbow Surg 6 (1997), 340-346.

[19] R.E. Glousman, F. Jobe and J. Tibone et al., Dynamic electromyographic analysis of the throwing with glenohumeral instability, J Bone Joint Surg 70(A) (1988), 220226.

[20] R.W. Gülch, Force-velocity relations in human skeletal muscle, Int J Sports Med 15 (1994), 2-10. 
[21] M. Järvinen, Epidemiology of tendon injuries in sports, Clin Sports Med 11 (1992), 493-504.

[22] M. Järvinen, Lower leg overuse injuries in athletes, Knee Surg Sports Traumatol Arthrosc 1 (1993), 126-130.

[23] K. JØrgensen and S. Bankov, Maximum strength of elbow flexors with pronated and supinated forearm, Med Sport 6 (1971), 174-180.

[24] K.L. Jensen and C.A. Rockwood, Shoulder arthroplasty in recreational golfers, $J$ Shoulder Elbow Surg 7 (1998), 362-367.

[25] P. Kannus, Isokinetic evaluation of muscular performance: implications for muscle testing and rehabilitation, Int J Sports Med 15 (1994), 11-18.

[26] J.F. Kramer, D. Nusca and L. Bisbee et al., Forearm pronation and supination : reliability of absolute torques and nondominant/dominant ratios, $J$ Hand Ther $\mathbf{7}$ (1994), 15-20.

[27] B.S. Kraushaar and R.P. Nirschl, Current concepts review tendinosis of the elbow (tennis elbow), J Bone Joint Surg 81(A-2) (1999), 259-278.

[28] LA. Levene, B.A. Hart and R.H. Seeds et al., Reliability of reciprocal isokinetic testing of the knee extensors and flexors, J Orthop Sports Phys Ther 14 (1991), 121127.

[29] D.G. Mandalidis and M. O’Brien, Isokinetic strength of the elbow flexors with the forearm in supination and in the neutral position, Isokinetics Exerc Sci 9 (2001), $111-117$.

[30] F. Mayer, T. Horstmann and U. Kranenbert et al., Reproducibility of isokinetic peak torque and angle at peak torque in the shoulder joint, Int J Sports Med 15 (1994), 2631. 
[31] L. Molczyk, L.K. Thigpen and J.A. Eickhoff et al., Reliability of testing the knee extensors and flexors in healthy adult women using a Cybex II Isokinetic Dynamometer, J Orthop Sports Phys Ther 14 (1991), 37-41.

[32] A.C. Rettig and D.V. Patel, Epidemiology of elbow, forearm, and wrist injuries in the athlete, Clin Sports Med 14 (1995), 289-297.

[33] AA. Sapega, Muscle performance evaluation in orthopedic practice. Journal of Bone and Joint Sugery 72A (1990), 1562-1574.

[34] W.D. Stanish, R.M. Rubinovich and S. Curwin, Eccentric exercise in chronic tendinitis, Clin Orthop Rel Res 208 (1986), 65-68.

[35] S.H. Westing and J.Y. Seger, Eccentric and concentric torque-velocity characteristics, torque output comparisons, and gravity effect torque corrections for the quadriceps and hamstring muscles in females, Int J Sports Med 10 (1989), 175-180. 


\section{TABLES LEGENDS}

Table 1 : CV (\%) for forearm pronator-supinator muscles and for the flexors and extensors of the wrist.

Table 2 : Mean values ( \pm 1 SD) of normalized peak-torques $\left(\mathrm{N} . \mathrm{m}_{\mathrm{kg}} \mathrm{kg}^{-1}\right.$ ) for the dominant and non-dominant pronator and supinator muscles in the male group.

Table 3 : Mean values ( \pm 1 SD) of normalized peak-torques $\left(\mathrm{N} . \mathrm{m} \cdot \mathrm{kg}^{-1}\right.$ ) for the dominant and non dominant sides pronator and supinator muscles in female group.

Table 4 : Mean values ( \pm 1 SD) of the PRO/SUP ratios in males and females.

Table 5 : Mean values ( $\pm 1 \mathrm{SD}$ ) of normalized peak-torques $\left(\mathrm{N} \cdot \mathrm{m}_{\mathrm{kg}} \mathrm{kg}^{-1}\right.$ ) for the dominant and non dominant flexor and extensor muscles in the male group.

Table 6 : Mean values ( $\pm 1 \mathrm{SD}$ ) of normalized peak-torques $\left(\mathrm{N} \cdot \mathrm{m}_{\mathrm{kg}} \mathrm{kg}^{-1}\right.$ ) for the dominant and non-dominant flexor and extensor muscles in the female group.

Table 7 : Mean values ( \pm 1 SD) of the FLE/EXT ratios in males and females. 


\section{FIGURES}

Fig. 1 : Subject installation for the forearm pro-supinator muscles assessment.

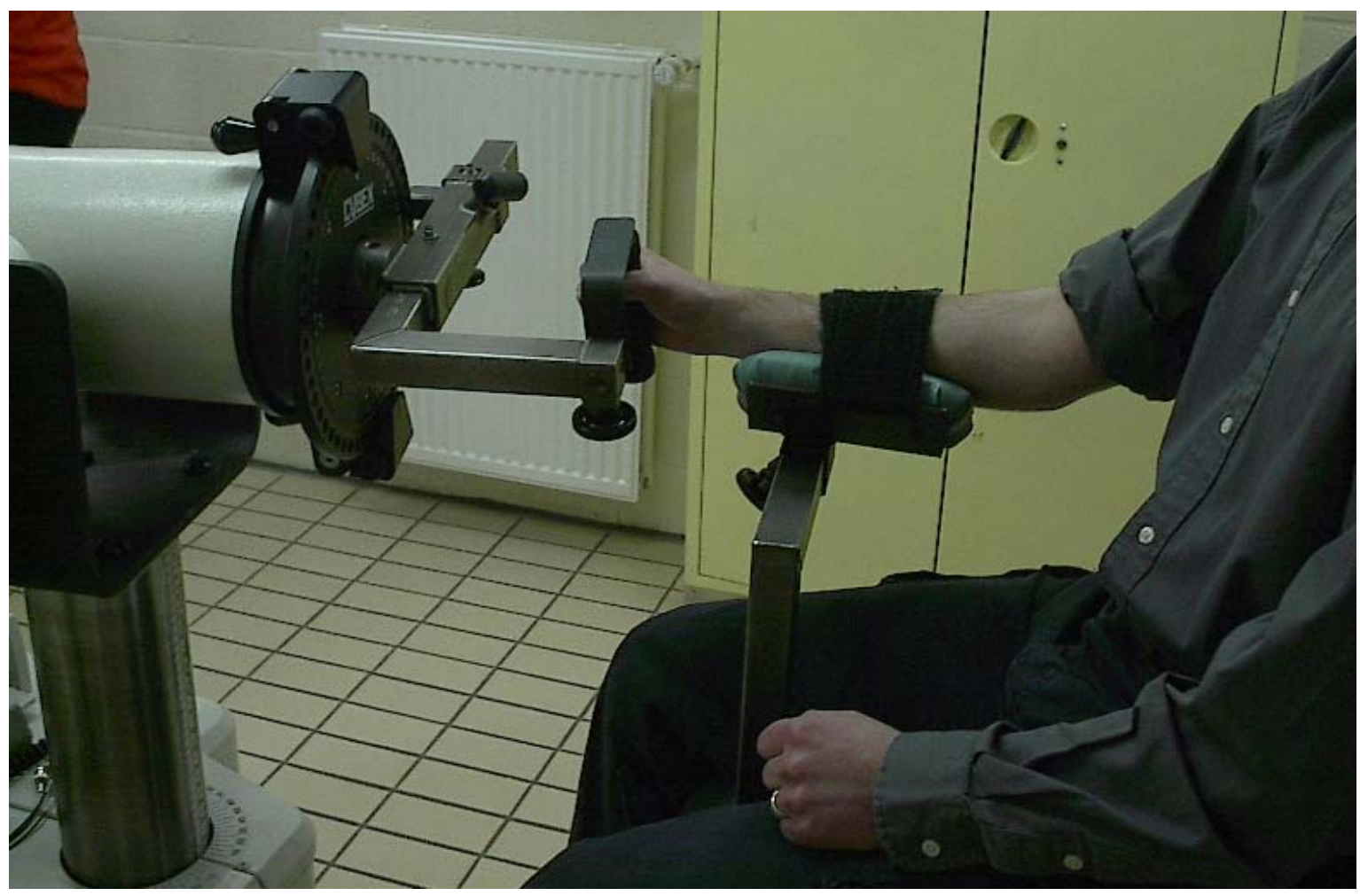

Fig. 2 : Subject installation for the wrist flexor - extensor muscles assessment.

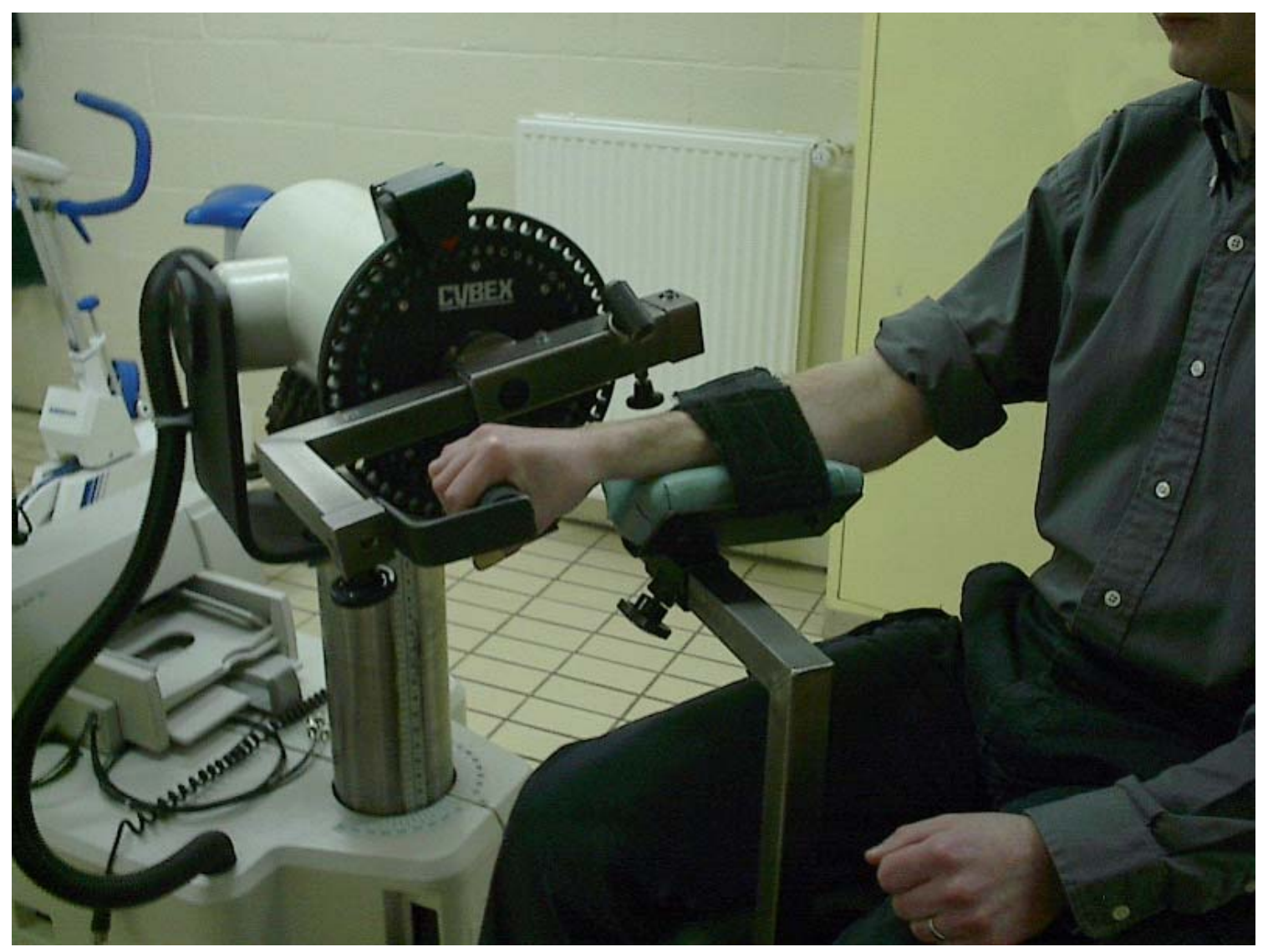


Table 1

MUSCLE GROUPS

TEST

$\begin{array}{llll}\text { PRO SUP } & \text { FLE }\end{array}$

C 90

C 30

10.0

11.7

E 60

2.0

11.6

6.6

6.2

4.9

9.4

10.3

11.0

5.6

6.9

1: C - concentric, E - eccentric, 30,60,90 - velocities

Table 2

\begin{tabular}{ccccccc}
\hline Muscles & Test & NPT - D & NPT -ND & p & PT - D & PT-ND \\
& & & & & & \\
& C90 & $0.14 \pm 0.03$ & $0.13 \pm 0.04$ & 0.127 & $10.5 \pm 3.6$ & $10 \pm 3.8$ \\
Pronators & C30 & $0.15 \pm 0.03$ & $0.16 \pm 0.03$ & 0.616 & $11 \pm 4$ & $11.8 \pm 4$ \\
(PRO) & E60 & $0.21 \pm 0.04$ & $0.18 \pm 0.05$ & $\underline{0.015}$ & $15 \pm 5.2$ & $13.6 \pm 6.7$ \\
\hline & & & & & & $8.6 \pm 2.1$ \\
Supinators & C30 & $0.15 \pm 0.02$ & $0.12 \pm 0.02$ & $\underline{0.012}$ & $10.9 \pm 2.3$ & $8 \pm 1.22$ \\
$($ SUP) & E60 & $0.20 \pm 0.04$ & $0.16 \pm 0.03$ & 0.069 & $15.6 \pm 5.1$ & $13.4 \pm 3.4$ \\
\hline
\end{tabular}

2: C - concentric, E - eccentric, 30,60,90 - velocities, PT - peak torque (Nm), NPT normalized peak Torque (Nm/kgbw), D- dominant, ND - nondominant. 
Table 3

\begin{tabular}{|c|c|c|c|c|c|c|}
\hline Muscles & Test & NPT - D & NPT -ND & $\mathbf{p}$ & PT - D & PT-ND \\
\hline \multirow{3}{*}{$\begin{array}{c}\text { Pronators } \\
\text { (PRO) }\end{array}$} & C90 & $0.09 \pm 0.01$ & $0.11 \pm 0.03$ & $\underline{0.035}$ & $5.5 \pm 1.3$ & $6.9 \pm 2.4$ \\
\hline & C30 & $0.11 \pm 0.02$ & $0.12 \pm 0.03$ & 0.697 & $7 \pm 2$ & $7.1 \pm 2.5$ \\
\hline & E60 & $0.14 \pm 0.03$ & $0.14 \pm 0.03$ & 0.541 & $8.4 \pm 2.3$ & $8.9 \pm 2.5$ \\
\hline \multirow{3}{*}{$\begin{array}{l}\text { Supinators } \\
\text { (SUP) }\end{array}$} & C90 & $0.10 \pm 0.02$ & $0.08 \pm 0.02$ & $\underline{0.01}$ & $6 \pm 1.2$ & $5 \pm 1.2$ \\
\hline & C30 & $0.11 \pm 0.02$ & $0.10 \pm 0.02$ & 0.146 & $6.6 \pm 1.4$ & $5.8 \pm 1.6$ \\
\hline & E60 & $0.15 \pm 0.03$ & $0.14 \pm 0.03$ & 0.373 & $9 \pm 1.5$ & $8.5 \pm 2.3$ \\
\hline
\end{tabular}

3: C - concentric, E - eccentric, 30,60,90 - velocities, PT - peak torque (Nm), NPT normalized peak Torque (Nm/kgbw), D- dominant, ND - nondominant.

Table 4

\begin{tabular}{ccccc}
\hline $\begin{array}{c}\text { Ratio } \\
\text { PRO/SUP }\end{array}$ & Test & D & ND & p \\
\hline & & & & \\
Male group & C 90 & $1.04 \pm 0.25$ & $1.12 \pm 0.36$ & 0.504 \\
& C 30 & $1.05 \pm 0.16$ & $1.33 \pm 0.33$ & $\underline{0.011}$ \\
\hline & E 60 & $1.04 \pm 0.16$ & $1.08 \pm 0.36$ & 0.751 \\
Female group & C 30 & $0.94 \pm 0.23$ & $1.45 \pm 0.42$ & $\underline{0.003}$ \\
& E 60 & $1.08 \pm 0.42$ & $1.22 \pm 0.25$ & 0.282 \\
\hline
\end{tabular}

4: C - concentric, E - eccentric, 30,60,90 - velocities, D- dominant, ND - nondominant. 
Table 5

\begin{tabular}{ccccccc}
\hline Muscles & Test & NPT - D & NPT - ND & p & PT - D & PT-ND \\
& & & & & & \\
& C90 & $0.35 \pm 0.04$ & $0.28 \pm 0.05$ & $\underline{0.000}$ & $26.7 \pm 4.5$ & $20.7 \pm 4.3$ \\
Flexors & C30 & $0.35 \pm 0.05$ & $0.29 \pm 0.05$ & $\underline{0.002}$ & $26.1 \pm 2.8$ & $21.5 \pm 3.8$ \\
(FLE) & E60 & $0.44 \pm 0.06$ & $0.34 \pm 0.06$ & $\underline{0.000}$ & $33.4 \pm 4.9$ & $26.1 \pm 4.2$ \\
\hline & & & & & $10.6 \pm 2$ & $10.5 \pm 1.8$ \\
Extensors & C30 & $0.15 \pm 0.03$ & $0.17 \pm 0.04$ & 0.078 & $11.3 \pm 2.3$ & $12.5 \pm 2.22$ \\
(EXT) & E60 & $0.23 \pm 0.08$ & $0.24 \pm 0.06$ & 0.541 & $17 \pm 4.6$ & $17.10 \pm 4.2$ \\
\hline
\end{tabular}

5: C - concentric, E - eccentric, 30,60,90 - velocities, PT - peak torque (Nm), NPT normalized peak Torque (Nm/kgbw), D- dominant, ND - nondominant.

Table 6

\begin{tabular}{ccccccc}
\hline Muscles & Test & NPT - D & NPT -ND & p & PT - D & PT-ND \\
& & & & & & \\
& C90 & $0.26 \pm 0.06$ & $0.22 \pm 0.07$ & 0.091 & $14.6 \pm 3.37$ & $13 \pm 4$ \\
Flexors & C30 & $0.28 \pm 0.08$ & $0.25 \pm 0.07$ & $\underline{0.036}$ & $15.7 \pm 4.3$ & $14.7 \pm 4$ \\
(FLE) & E60 & $0.35 \pm 0.11$ & $0.30 \pm 0.09$ & 0.159 & $19.3 \pm 5.7$ & $18.1 \pm 5$ \\
\hline & & & & & & $7.6 \pm 1.4$ \\
Extensors & C30 & $0.13 \pm 0.02$ & $0.14 \pm 0.03$ & 0.216 & $7.6 \pm 1.6$ & $8.6 \pm 2.3$ \\
(EXT) & E60 & $0.28 \pm 0.06$ & $0.25 \pm 0.04$ & 0.164 & $15.4 \pm 3.6$ & $14.6 \pm 2$ \\
\hline
\end{tabular}

6: C - concentric, E - eccentric, 30,60,90 - velocities, PT - peak torque (Nm), NPT normalized peak Torque (Nm/kgbw), D- dominant, ND - nondominant. 
Table 7

\begin{tabular}{ccccc}
\hline $\begin{array}{c}\text { Ratio } \\
\text { FLE/EXT }\end{array}$ & Test & D & ND & p \\
\hline & & & & \\
Male group & C 90 & $2.55 \pm 0.29$ & $1.99 \pm 0.40$ & $\underline{0.001}$ \\
& C 30 & $2.38 \pm 0.43$ & $1.77 \pm 0.52$ & $\underline{0.008}$ \\
& E 60 & $2.02 \pm 0.53$ & $1.59 \pm 0.39$ & $\underline{0.005}$ \\
\hline Female group & C 30 & $2.16 \pm 0.63$ & $1.93 \pm 0.90$ & 0.393 \\
& E 60 & $1.23 \pm 0.28$ & $1.27 \pm 0.28$ & $\underline{0.012}$ \\
\hline
\end{tabular}

7: C - concentric, E - eccentric, 30,60,90 - velocities, D- dominant, ND - nondominant. 